\title{
Improvement of the retention of ocimene in water phase using Class II hydrophobin HFBII
}

\author{
Mohammadreza Khalesi, ${ }^{\text {a }}$ Nathalie Mandelings, ${ }^{a}$ Beatriz Herrera-Malaver, ${ }^{\text {a,b }}$ \\ David Riveros-Galan, ${ }^{a}$ Kurt Gebruers ${ }^{a}$ and Guy Derdelinckx ${ }^{a}$
}

\begin{abstract}
Hydrophobins have exceptional surface activity with potential applications for food and pharmaceutical industry. In this study, the effect of Class II hydrophobin HFBII on the retention of a volatile compound, ocimene in a water phase was investigated. We observed a negative gushing when $200 \mu$ g pure HFBII ( $\kappa$-grade) and $40 \mu \mathrm{g}$ ocimene (assay $\geq 90 \%$ ) was added to $1 \mathrm{~L}$ sparkling water with $\mathrm{CO}_{2}$ concentration of $7 \mathrm{~g} / \mathrm{L}$. The droplet size analysis of $\kappa-\mathrm{HFBII}$, ocimene and the mixture of them showed that the

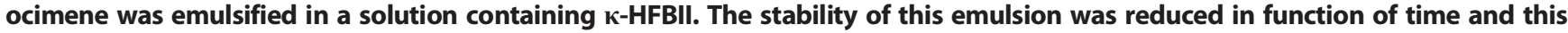
effect was intensified by increasing the temperature and the presence of $\mathrm{CO}_{2}$. Additionally, the retention of ocimene in a $\mathrm{HFBII}$ rich solution was measured by SPME-GC-MS. When $0.26 \pm 0.03 \mathrm{mg} \mathrm{HFBII} / \mathrm{mL}$ is added to ocimene solution, $43 \%$ of the original ocimene concentration (i.e. $5.9 \times 10^{-6} \mathrm{mg} / \mathrm{mL}$ ) remained in the solution after 3 days, and $32 \%$ after 7 days. In contrast, original ocimene concentration in solutions without HFBII was only retained $20 \%$ and $14 \%$, respectively. It showed the potential of HFBII to incorporate insoluble flavour compounds in beverages and control their release. Copyright $\odot 2015$ John Wiley \& Sons, Ltd.
\end{abstract}

Additional supporting information may be found in the online version of this article at the publisher's web site

Keywords: hydrophobin; ocimene; gushing; GC-MS; retention; aroma

\section{Introduction}

Flavour stability has been of increasing interest due to its relationship with the quality and the acceptability of products. However, the flavour stability is difficult to control since it is directly related to the existence of different ingredients in the system. ${ }^{[1]}$ The presence of oil phases, proteins and carbohydrates are the keys to control the stability. For instance, polysaccharides reduce the volatility of aroma compounds by increasing the viscosity, and by specific molecular interactions. ${ }^{[2]}$ On the other hand, proteins may result in an increased retention of aroma compounds in the liquid phase by encapsulation. ${ }^{[3]}$ Encapsulation of flavour compounds, especially monoterpenes, is a technique to protect them from undesirable effects such as light induced reactions, oxidation and reactions with other components in food matrix. ${ }^{[4]}$ The retention capacity is mainly associated with the physicochemical characteristics of the encapsulant like molecular weight (MW), molecular conformation and chemical functionality. The encapsulants include carbohydrates, gums, cellulose and derivatives, lipids, and some proteins. ${ }^{[5]}$ In general, aroma compounds can be bound to the proteins through hydrogen bonds or hydrophobic interaction, making them less volatile. ${ }^{[6]}$

Hydrophobins are amphiphilic proteins with a potential for encapsulating and resolving hydrophobic molecules in aqueous environments. These characteristics are based on their ability to form highly ordered monolayers at hydrophilic/hydrophobic interfaces via self-assembly. ${ }^{[7-9]}$ Class II hydrophobins contain one or more hydrophobic patch(es), suggesting important applications in the food and pharmaceutical industry. ${ }^{[10]}$ The presence of the hydrophobic patch in hydrophobins proposes an interaction with apolar components (e.g. essential oils and other aroma compounds), consequently increasing their retention for longer periods of time.
The aim of this work was to investigate the possibility of using Class II hydrophobins to prolong the retention of aroma compounds in a solution. As proof of principle, the retention of a monoterpene in presence of Class II hydrophobin HFBII was tested. Monoterpenes $\left(C_{10}\right)$ are neutral organic molecules with low solubility in water and high volatility. ${ }^{[11]}$ Many of them are used for seasoning and flavouring food, perfume and other household items. ${ }^{[12]}$ For instance, ocimene and myrcene are two hydrophobic monoterpenes with the same molecular formula $\left(\mathrm{C}_{10} \mathrm{H}_{16}\right)$ and are mostly used in fragrances. Both molecules are derived from geranyl diphosphate. ${ }^{[13]}$ Myrcene can be found within cannabis, lemon grass and hops. ${ }^{[14]}$ On the other hand, ocimene with its warm-herbaceous, citrus, tropical, green, woody odour is very diffusive with relatively low tenacity. Ocimene can be found in hop oil and is a typical aroma in carbonated beverages. ${ }^{[15,16]}$ Without use of any emulsifier or encapsulating agent, ocimene will gradually migrate to the interface of the liquid and escape to the gas phase as many other aroma compounds. In this study, ocimene was selected as a model aroma compound to measure the capacity of hydrophobins for retaining the aroma in beverages. HFBII, the first hydrophobin with an entirely determined structure, ${ }^{[17]}$ was also used as a model of Class II hydrophobins.

\footnotetext{
* Correspondence to: Mohammadreza Khalesi, Department of Microbial and Molecular Systems (M2S), KU Leuven, B-3001, Heverlee, Belgium. E-mail: m.khalesi@ag.iut.ac.ir

a Department of Microbial and Molecular Systems $\left(M^{2} S\right)$, KU Leuven, B-3001 Heverlee, Belgium

b VIB Laboratory of Systems Biology, Gaston Geenslaan 1, B-3001 Heverlee, Belgium
} 
During this study, first the possibility of interaction between ocimene and HFBll was determined by gushing test. Gushing phenomenon in carbonated beverages is the spontaneous and wild overfoaming at bottle opening. This occurs as a result of the interaction of $\mathrm{CO}_{2}$ molecules with the hydrophobic patches of the elastic Class II hydrophobin films. ${ }^{[18]}$ The interaction between ocimene and HFBII would occupy the hydrophobic patch of HFBII, thus inhibiting gushing by disrupting or preventing the $\mathrm{CO}_{2}-\mathrm{HFBII}$ interaction. Moreover, a droplet size analysis was performed to see whether new droplets formed after mixing HFBII and ocimene. The next step was to apply this potential as a positive function. For this purpose, the retention of ocimene in a water phase when adding $\kappa$-HFBII was measured by direct immersion solid phase microextraction (DI-SPME) followed by gas chromatography mass spectrometry (GC-MS). We demonstrated the potential of Class II hydrophobins to increase the retention time of the flavour compounds in the water phase.

\section{Experimental}

\section{Production, purification and verification of hydrophobin HFBII}

Trichoderma reesei MUCL 44908 (purchased from BCCM/MUCL (Agro)Industrial Fungi \& Yeast Collection, Louvain-la-Neuve, Belgium) was cultivated in a $1 \mathrm{~L}$ working volume fermenter (KGW-Isotherm, Schieder $\mathrm{GmbH}$, Karlsruhe, Germany) equipped with an agitator (RW20 digital, IKA GmbH, Staufen, Germany) at $29^{\circ} \mathrm{C}$ for 5 days as the actual time of fermentation. The composition of aqueous medium is presented in Table 1.

Phosphoric a cid was used to adjust the $\mathrm{pH}$ to $4.5-5.0 .^{[19]}$ For inoculation, the spores were collected from the surface of $T$. reesei cultures that grow on malt extract agar (MEA) (Merck KGaA, Darmstadt, Germany) in petri dishes, and placed into test tubes containing $10 \mathrm{~mL}$ fresh medium culture. The overnight culture was added to the fresh medium (Table 1). After 5 days of fermentation, the mycelium was discarded by centrifugation $(8000 \mathrm{~g}$ for 25 min at $6{ }^{\circ} \mathrm{C}$, Beckman model J2-21, Brea, USA) and the supernatant was stored at $2{ }^{\circ} \mathrm{C}$ for further analysis.

The supernatant was subjected to a foam fractionation system for prepurification. The foam fractionation device consisted of a glass column with internal diameter (ID) of $52 \mathrm{~mm}$. For foaming, the gaseous $\mathrm{CO}_{2}$ passed through a sintered glass disk with the liquid containing HFBII $(\mathrm{pH}=4.7)$. This step was followed at room temperature. The liquid did not pass through the sintered glass. After collecting the foamate, centrifugation $(8000 \mathrm{~g}$ for 5 min at $25^{\circ} \mathrm{C}$, Beckman model J2-21, Brea, USA) was performed to collapse the foam. The resulting sample is further referred to as $\alpha_{\mathrm{L}}$-HFBII. The gas flow rate of $3 \mathrm{~L} / \mathrm{min}$, ratios of the liquid height over the column height of

Table 1. Ingredients for production of hydrophobin HFBII by T. reesei

\begin{tabular}{|c|c|}
\hline Component & Amount ( $\mathrm{g} / \mathrm{L})$ \\
\hline Lactose & 40.0000 \\
\hline Peptone & 4.0000 \\
\hline Yeast Extract & 1.0000 \\
\hline $\mathrm{KH}_{2} \mathrm{PO}_{4}$ & 4.0000 \\
\hline$\left(\mathrm{NH}_{4}\right)_{2} \mathrm{SO}_{4}$ & 2.8000 \\
\hline $\mathrm{MgSO}_{4} \cdot 7 \mathrm{H}_{2} \mathrm{O}$ & 0.6000 \\
\hline $\mathrm{CaCl}_{2} \cdot 2 \mathrm{H}_{2} \mathrm{O}$ & 0.8000 \\
\hline $\mathrm{FeSO}_{4} \cdot 7 \mathrm{H}_{2} \mathrm{O}$ & 0.0100 \\
\hline $\mathrm{CoCl}_{2} \cdot 6 \mathrm{H}_{2} \mathrm{O}$ & 0.0040 \\
\hline $\mathrm{MnSO}_{4} \cdot \mathrm{H}_{2} \mathrm{O}$ & 0.0032 \\
\hline $\mathrm{ZnSO}_{4} \cdot 7 \mathrm{H}_{2} \mathrm{O}$ & 0.0069 \\
\hline
\end{tabular}

0.33 , and glass filter $G_{3}$ (pore size $=15-40 \mu \mathrm{m}$ ) were used as the optimal separation conditions for $\mathrm{CO}_{2}$ foam fractionation. ${ }^{[20]}$

After obtaining $\alpha_{\mathrm{L}}$-HFBll, purification with reversed phase liquid chromatography (RP-LC) was carried out using a Source 30RPC resin (GE Healthcare; Uppsala, Sweden). By using RP-LC (Bio-Rad laboratories n.v., Eke, Belgium), elution of proteins was carried out with a linear gradient of acetonitrile $(\mathrm{ACN})$ in MilliQ water containing $0.1 \%$ trifluoroacetic acid (TFA) (from 0 to $60 \% \mathrm{ACN}$ ) at a flow rate of $10 \mathrm{~mL} / \mathrm{min}$ and was monitored by UV detection at $214 \mathrm{~nm}$.

Identification of hydrophobins was performed by matrix assisted laser desorption/ionization time of flight (MALDI-TOF) with an Ultraflex II instrument in linear mode (Brüker Daltonics, GmbH, Bremen, Germany) using $\alpha$-cyano-4-hydroxy cinnamic acid to form the matrix. The fractions collected from chromatography were dried in a vacuum centrifuge (Univapo 150 Ech and Multitrap, Montreal, Canada) and re-dissolved in $100 \mu \mathrm{L}$ MilliQ water with $5 \%$ ACN containing $0.5 \%$ formic acid (FA). The samples were vortexed with a bench mixer ( $30 \mathrm{~s}, 1000 \mathrm{rpm}$ ) and sonicated for $5 \mathrm{~min}$ in an ultrasonic bath (Branson 2510, Wareham, USA) to avoid aggregation. $10 \mathrm{mg}$ of $\alpha$-cyano-4-hydroxy cinnamic acid was added to $200 \mu \mathrm{L}$ MilliQ water containing $50 \% \mathrm{ACN}$ and $0.5 \% \mathrm{FA}$, and then sonicated for $5 \mathrm{~min} .1 \mu \mathrm{L}$ of the sample was placed on the target plate (MTP 384 ground steel, Brüker Daltonics, $\mathrm{GmbH}$, Bremen, Germany) and mixed with $1 \mu \mathrm{L}$ matrix solution. The plate was then placed in the MALDI-TOF apparatus and a measurement was carried out in the range of $0-40 \mathrm{kDa}$. The fractions containing only proteins with MW in the range of 7.0-7.2 kDa were considered as HFBll samples.

After confirmation of MW with MALDI-TOF, the amount of HFBll present in those samples were quantified using a micro-spectrophotometer (NanoDrop ND-1000, Wilmington, USA). The wavelength was adjusted to $280 \mathrm{~nm}$ and the extinction coefficient $(\varepsilon)$ to 10 . The blank was considered to be a solution of $40 \%$ ACN containing $0.1 \%$ TFA corresponding to the mobile phase at the elution time of HFBII by chromatography.

\section{Gushing test}

Before following the retention profile of ocimene in the presence of hydrophobins, the possible interaction between ocimene and HFBII had to be proven. Upon addition of ocimene to positive gushing samples, the interaction between ocimene and HFBll can be determined by the gushing analysis. Through the possible interaction between ocimene and the hydrophobic patch of hydrophobin HFBII via hydrophobic interaction, the patch will no longer be free to interact with $\mathrm{CO}_{2}$ molecules, with the prohibition of gushing as a result.

Glass bottles of $1 \mathrm{~L}$ sparkling water with a $\mathrm{CO}_{2}$ concentration of $7.0 \mathrm{~g} / \mathrm{L}$ were stored at a temperature of $2{ }^{\circ} \mathrm{C}$ prior to the addition of HFBII. $1 \mathrm{~mL}$ of $\kappa-H F B I I ~(0.2 \mathrm{mg} / \mathrm{mL})$, that has been purified from chromatography using $40 \%$ ACN containing $0.1 \%$ TFA, was then added to each bottle. HFBll acts as a primary gushing inducer via the formation of $\mathrm{CO}_{2}$ stabilized nanobubbles. At opening, this will lead to an explosive overfoaming. Four different volumes $(0.01,0.05,0.1$ or $0.5 \mu \mathrm{L}$ ) of pure ocimene (assay $\geq 90 \%$, Sigma-Aldrich, Bornem, Belgium) were added to the samples. The bottles were then shaken horizontally in a shaking incubator (TH 30, Edmund Bühler, GmbH, Hechingen, Germany) for $72 \mathrm{~h}$ at $125 \mathrm{rpm}$ and $25^{\circ} \mathrm{C}$. Afterwards, the bottles were weighed and opened at $25{ }^{\circ} \mathrm{C}$ in accordance to a standard procedure. ${ }^{[18]}$ After observation of potential overfoaming, the bottles were reweighed, so that the mass difference was considered as the gushing volume. ${ }^{[18]}$ The positive control for this experiment was considered to be a sample only treated with $1 \mathrm{~mL} \kappa-\mathrm{HFBII}(0.2 \mathrm{mg} / \mathrm{mL})$ and the negative control was $1 \mathrm{~mL}$ solution of $40 \% \mathrm{ACN}$ with $0.1 \%$ TFA containing $1.0 \mu \mathrm{L}$ ocimene.

\section{Analysis of the droplet size}

The possibility of the interaction between hydrophobins and apolar molecules was analysed by droplet size analysis. The size of droplets in liquid samples were measured using a particle size analyser (Nano-flex 180, Microtrac, Meerbusch, Germany) which detects particles in the range of 0.8-6500 nm. 
In order to check whether the new droplets are created after mixing the ocimene and HFBII, both were separately analysed with particle size analyser and then the mixture was submitted to the system. The droplets for the samples containing ocimene are the micelles formed by gathering the molecules of ocimene. In the case of pure hydrophobin, the droplets are the bubbles covered by protein. In the case of mixture, both types of droplets are present. The changes in size of the droplets in the mixture showed the possible interaction between them.

Sample preparation:

Sample 1: $4 \mathrm{~mL} \kappa-H F B$ II with a concentration of $0.1 \mathrm{mg} / \mathrm{mL}$ was vortexed for $1 \mathrm{~min}$.

Sample 2: $0.1 \mu \mathrm{L}$ ocimene diluted in $4 \mathrm{~mL}$ solvent (ACN $40 \%$ ) was vortexed for $1 \mathrm{~min}$.

Sample 3: $4 \mathrm{~mL}$ of $\kappa$-HFBll with a concentration of $0.1 \mathrm{mg} / \mathrm{mL}$ was mixed with $0.1 \mu \mathrm{L}$ ocimene and vortexed for $1 \mathrm{~min}$.

All the samples were analysed with particle size analyser in order to measure the droplet size. Two replicates were performed per type of samples. The evolution of the particle size in the samples were measured every minute during 30 minutes. To ensure the stability of the obtained emulsion, the same measurement procedure was carried out again after 4, 8 and 10 days.

\section{Migration of HFBII to the surface}

$1 \mathrm{~mL} \kappa-H F B l l$ (with a concentration of $2 \mathrm{mg} / \mathrm{mL}$ ) and ocimene $(0,0.1 \mu \mathrm{L}$ or $0.5 \mu \mathrm{L}$ ) were vortexed with a speed of 1000 and 2000 rotations/min or mixed using a magnet field apparatus ${ }^{[21]}$ for $15 \mathrm{~min}$ and then added to decanters containing $100 \mathrm{~mL}$ liquid (distilled water or carbonated water with $7 \mathrm{~g} \mathrm{CO}_{2} / \mathrm{L}$ ). The closed decanters were stored at the temperatures of $2{ }^{\circ} \mathrm{C}, 25^{\circ} \mathrm{C}$ or $40^{\circ} \mathrm{C}$. The hypothesis is that since the HFBll molecules cover the ocimene micelles, the rate of HFBII migration to the surface is reduced. This was examined by the determination of the concentration of HFBII in the upper $10 \%$ of the liquid (labelled on the decanter) after 1, 3 and 7 days of storing by using a micro-spectrophotometer (NanoDrop ND-1000; Wilmington, USA) adjusted on wavelength $280 \mathrm{~nm}$ and $\varepsilon=10 .{ }^{[18,22]}$ Each experiment here was performed with one variable and several constants. The rate of HFBII migration at different conditions was therefore obtained.

\section{GC-MS analysis}

A Direct Immersion Solid Phase Micro Extraction Gas Chromatography Mass Spectroscopy (DI-SPME-GC-MS) was used to track the retention time of ocimene in a solution. ${ }^{[23]}$

$2.5 \mathrm{~mL}$ from a solution of ocimene with concentration $8.2 \times 10^{-6} \mathrm{mg} / \mathrm{mL}$ (mixture of isomers assay $\geq 90 \%$ ) was added to a glass vial and then $1 \mathrm{~mL}$ of $\kappa-H F B I I ~(0.07 \pm 0.02 \mathrm{mg} / \mathrm{mL}$ or $0.9 \pm 0.1 \mathrm{mg} / \mathrm{mL})$ was supplemented. The final concentrations of HFBII in the required volume $(3.5 \mathrm{~mL})$ were equal to $0.020 \pm 0.006 \mathrm{mg} / \mathrm{mL}$ and $0.260 \pm 0.030 \mathrm{mg} / \mathrm{mL}$, respectively. The samples were vortexed for $1 \mathrm{~min}$ and then transferred into vials of $4 \mathrm{~mL}$. $2 \mu \mathrm{L}$ internal standard (linalool 97\%, Sigma-Aldrich, St. Louis, USA) with a concentration of $4.35 \times 10^{-3} \mathrm{mg} / \mathrm{mL}$ with $\mathrm{NaCl}(25 \% \mathrm{w}: \mathrm{v})$ were added to the vial. For extraction, a polydimethylsiloxane (PDMS) fibre (100 $\mu$ L PDMS, Supelco Co., Bellafonte, USA) was used. The SPME fibre was completely immersed in the samples in the $4 \mathrm{~mL}$ vial. The PDMS fiber was exposed to the solution for $15 \mathrm{~min}$ during which the samples were stirred. After the sampling time, the compounds trapped on the fibre were thermally desorbed in the injection port of a gas chromatograph mass spectrometer (Shimadzu QP2010 GC Ultra-MS, Shimadzu, s-Hertogenbosch, Netherlands). The instrument was controlled by GCMS solutions software and was equipped with an HP-5ms non-polar column (Agilent, $30 \mathrm{~m} \times 0.25 \mathrm{~mm}$, $\mathrm{ID}=0.25 \mu \mathrm{m}$ thin layer). Helium was used as carrier gas and constant pressure of $140 \mathrm{kPa}$. The injection was at split mode with a ratio of 1:100. The temperature programme used started at $40{ }^{\circ} \mathrm{C}$ and rose to $220^{\circ} \mathrm{C}$ at a rate of $20^{\circ} \mathrm{C} / \mathrm{min}$. The injection port and detector temperatures were kept at $250^{\circ} \mathrm{C}$. All the steps were performed after 3 and 7 days to track the stability of the ocimene in the mixture with 2 repetitions. The mass detector was operated in scan mode (35-600 amu) using electronic impact ionization (70 eV).
The concentration of ocimene in solution was calculated using a calibration curve. Five samples of $2.5 \mathrm{ml}$ were prepared in $4 \mathrm{~mL}$ vials, containing different concentration of ocimene $(0.3,0.6,1.8,2.9$, or $4.1 \mathrm{ng} / \mathrm{mL})$. Again $2 \mu \mathrm{L}$ of a $4.35 \times 10^{-3} \mathrm{mg} / \mathrm{mL}$ linalool $(97 \%$, Sigma-Aldrich, St. Louis, USA) was added as internal standard as well as $\mathrm{NaCl}(25 \%$ w:v). The amount of ocimene per $3.5 \mathrm{~mL}$ (total volume) was plotted with respect to the relative peak area of ocimene over the internal standard.

\section{Results and discussion}

\section{Characterization of hydrophobin HFBII}

The production and the purification methods for hydrophobins from Trichoderma reesei, HFBI and HFBII, are different. To produce HFBII addition of lactose to the system is needed, though glucose is essential to produce HFBI. In the case of HFBll production, foam fractionation system can be used due to the fact that the protein released to the medium, while HFBI remains on the mycelium and for such a protein, use of foam fractionation is not applied. Actually, HFBII is produced to protect spores when the fungi is faced with starvation condition. $\mathrm{HFBI}$ on the other hand is produced in order to help fungi to move from a hydrophilic environment to a hydrophobic one, and vice versa. ${ }^{[20]}$ Final purification of HFBI and HFBII using chromatography follow however the same procedure.

An example of the chromatograms generated during purification is shown in Figure 1. The fractions of interest were eluted at 35-50\% ACN.

The chromatography fractions were submitted to the MALDITOF system to check the purity of the final product. After analysis of several collected fractions using MALDI-TOF, the molecular weight of the proteins in the range of $0-40 \mathrm{kDa}$ was displayed. The MW equal to $7.042 \mathrm{kDa}$ was recorded in few fractions (refer to supplementary data). This MW corresponds to the intact mature HFBII molecule minus the last amino acid (Phenylalanine ${ }_{71}$ ) ${ }^{[24]}$ The loss of the last amino acid could be explained by biodegradation at the end of the stationary phase of the moulds, as a similar occurrence has also been observed for mycotoxins. ${ }^{[2,25]}$ The important issue is whether losing the Phenylalanine ${ }_{71}$ affects the activity of HFBII or not. The hydrophobic sites of HFBII include 19 residues: Glycine $_{6}$, Leucine ${ }_{7}$, Leucine 12, Valine $_{18}$ to Valine 24 , Valine ${ }_{54}$ to Alanine $_{58}$, and Alanine 61 to Cysteine $_{64}$. These govern the main surface active properties of hydrophobins. Since Phenylalanine ${ }_{71}$ is located in the hydrophilic side of the protein, it does not change the activity of HFBII (refer to supplementary data). The positive

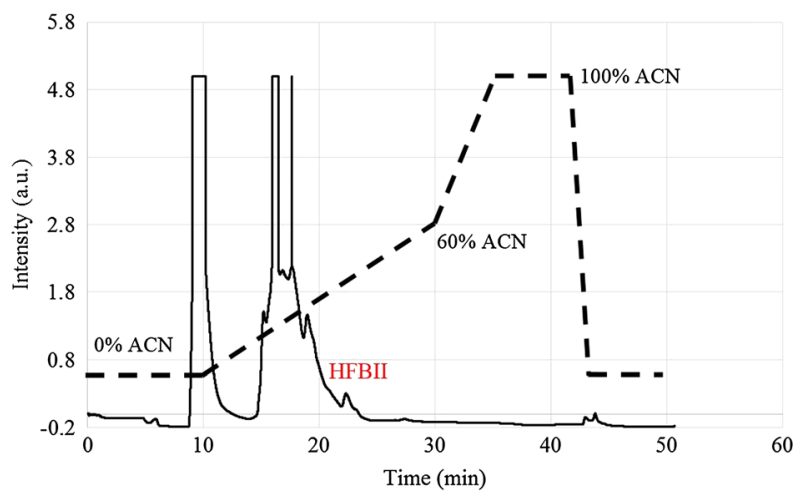

Figure 1. Chromatogram for the culture medium containing hydrophobin HFBll resulting from the fermentation of $T$. reesei after purification by $15 R P C$ column, recorded at a wavelength of $214 \mathrm{~nm}$. The dashed line represents the ACN\% gradient 
gushing using $18 \mu \mathrm{g} / \mathrm{L}$ truncated form of HFBll confirmed the activity of the obtained HFBII. ${ }^{[18]}$

The concentration of HFBII in the fermented liquid was $25 \pm 2$ $\mathrm{mg} / \mathrm{L}$. This was obtained after the collection of fractions related to HFBII from the chromatography and the measurement of the pure fractions (confirmed by MALDI-TOF) with NanoDrop. For samples after foam fractionation, the concentration of HFBll increased up to a total concentration of $115 \pm 8 \mathrm{mg} / \mathrm{L}$. Thus, the volumetric enrichment value for HFBII was equal to $4.6\left(=\frac{115 \mathrm{mg} / \mathrm{L}}{25 \mathrm{mg} / \mathrm{L}}\right)$.

\section{Gushing inhibition}

The possible interaction between HFBII and ocimene was measured by performing the gushing test. In gushing positive samples, $\mathrm{CO}_{2}$ and HFBII find each other quickly and create stable $\mathrm{CO}_{2}$ nanobubbles with potential for liquid expulsion after pressure release (bottle opening). If a molecule can compete with $\mathrm{CO}_{2}$ to block the hydrophobic patch of HFBII and prevent the occurrence of $\mathrm{CO}_{2}-\mathrm{HFBll}$ interaction, then it can inhibit primary gushing. Recently, it has been shown that the overfoaming is inhibited by addition of some linear saturated and unsaturated hydrocarbons or some fatty acids to the primary gushing positive beverages. ${ }^{[26]}$

Inhibition of gushing was an indication for the presence of an interaction between the hydrophobic patch of HFBll and ocimene. In Figure 2, the gushing results are depicted when adding different amounts of ocimene to $1 \mathrm{~L}$ of sparkling water contaminated by HFBII. Each bar gives the average value of a group. The first bar is the negative control which did not contain HFBll but only ocimene $(800 \mu \mathrm{g})$. Gushing was not observed for this sample. The second bar is the positive control which did not contain any ocimene but only HFBII $(0.2 \mathrm{mg})$ and it gushed. Although the variation in measuring the amount of overfoaming can be substantial (up to $50 \%$ relative standard deviation), it was still possible to distinguish between gushing and non-gushing samples. The $50 \%$ relative standard deviation observed in gushing results is due to the problem of gushing experiment. Up to now, a quantitative relation between the amount of gushing and the amount of hydrophobins is not available. Sarlin et al. (2005) ${ }^{[27]}$ showed indeed that identical quantities of different Class II hydrophobins added to identic reference bottles of sparkling

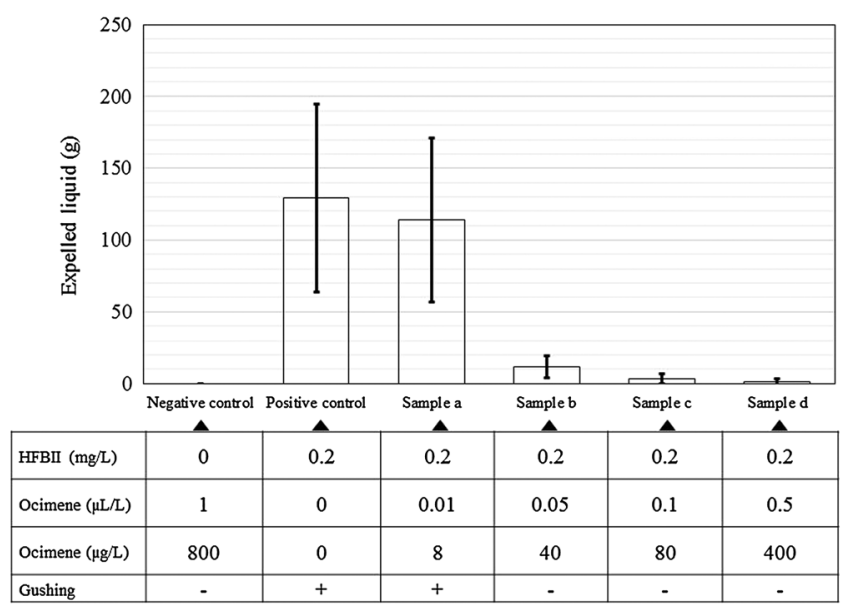

Figure 2. Expelled liquid ( $\mathrm{g} / \mathrm{L}$ ) observed after the gushing analysis. The concentration of ocimene and the gushing response for each treatment are depicted in the table. All the samples were prepared by addition of $0.2 \mathrm{mg} \kappa$-HFBll to induce gushing $(n=3)$. $\kappa$-HFBll was not added to the negative control water can provoke different quantities of overfoaming. Further, the absolute and scientific interpretation of the results is difficult and sometimes unreliable. Finally inter-laboratory tests to assess the gushing risk of samples provide divergent results.

The addition of ocimene with a concentration of $40 \mu \mathrm{g} / \mathrm{L}$ or higher stopped gushing induced by $0.2 \mathrm{mg} / \mathrm{L} \kappa$-HFBII. It can be concluded that ocimene interacts with the hydrophobic patch of $\mathrm{HFBIl}$, thus the critical step for creating the $\mathrm{CO}_{2}$ nanobubbles is avoided. ${ }^{[18]}$ There should be a threshold of ocimene present in the sample to occupy the hydrophobic patch, so that the patch no longer interacts with $\mathrm{CO}_{2}$. Hydrophobin HFBII has a hydrophobic patch with a surface accessible area (SAA) of $7.4 \mathrm{~nm}^{2}$. ${ }^{[28]}$ On the other hand, ocimene may interact with this patch horizontally, vertically or any orientation in between, or a combination of the above mentioned options. The width of an ocimene molecule was estimated to be $6.64 A^{\circ}$, the length $9.91 A^{\circ}$ (Marvin 6.1.0, 2013). The rough estimation number of ocimene molecules exposed to the hydrophobic SAA of HFBII equals to 11.25. The experimental results strongly support the calculations above showing that $40 \mu \mathrm{g}$ of ocimene $\left(1.77 \times 10^{17}\right.$ molecules, molar mass $\left.=136.2 \mathrm{~g} / \mathrm{mol}\right)$ is necessary to inactivate $0.2 \mathrm{mg} \mathrm{HFBll}\left(1.67 \times 10^{16}\right.$ molecules, molar mass $=7189 \mathrm{~g} / \mathrm{mol})$. In other words, 10.6 molecules of ocimene can interact with one molecule of HFBII in the practical experiment.

\section{Droplet size analysis}

The measurement of droplet sizes in a mixture of HFBll-ocimene and the comparison with the droplet size of each component individually, revealed a possible interaction between the components. An average distribution of 30 measurements of the droplet size (each measurement had a duration of $1 \mathrm{~min}$ ) for each sample of HFBII, ocimene and mixture was reported (Figure 3).

The results showed that probably an interaction between hydrophobin and ocimene occurred. Droplets of ocimene had an
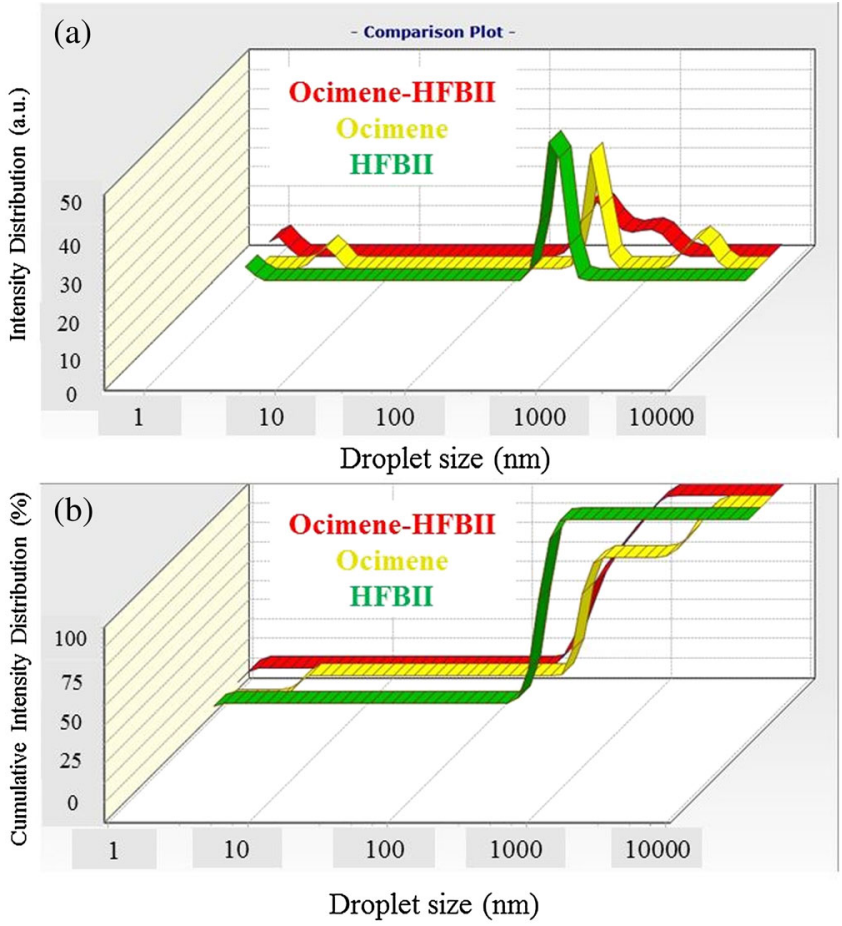

Figure 3. (a) Average diameter of the droplets, and (b) Cumulative intensity size distribution of ocimene, HFBll and the mixture of both 
average diameter size of $5 \mathrm{~nm}$ (present in very low amounts), 600 $\mathrm{nm}$ or values between $5500 \mathrm{~nm}$ to $6500 \mathrm{~nm}$ in solutions without HFBII. Probably there were bigger droplets in the samples, but the highest limit of detection for the system was $6500 \mathrm{~nm}$.

On the other hand, two peaks were observed in the solution with only $\kappa$-HFBII. The first peak showed droplets with an average of 2-3 nm diameter, which matches with the size of monomeric HFBII. The second peak represented the droplets with an average diameter size of $230 \mathrm{~nm}$. The latter might be related to the balanced air (or nitrogen) nanobubbles covered by hydrophobin HFBII in the sample after shaking, since the HFBII sample before shaking represented only the diameter of the monomeric model. In recent studies, it has been reported that stable nitrogen nanobubbles have a spherical shape with a diameter in the range of 100 to $434 \mathrm{~nm} \cdot{ }^{[29,30]}$ However, more evidence should be obtained before this hypothesis can be established.

When HFBII and ocimene were added together, new droplet sizes were observed. These droplets were bigger than $230 \mathrm{~nm}$ and smaller than $5500 \mathrm{~nm}$, suggesting that HFBll kept the ocimene droplets in smaller size, i.e. it emulsified the ocimene by (partially) covering the ocimene (Figure 4).

The evolution of the droplet size was tracked in function of time (during $30 \mathrm{~min}$, intervals of $1 \mathrm{~min}$ ). In the samples of $\kappa-\mathrm{HFBll}$, the same distribution in droplet size was observed during $30 \mathrm{~min}$. In the case of ocimene the droplets were around $600 \mathrm{~nm}$ and $2000 \mathrm{~nm}$ at the beginning. Over time, the latter aggregated quickly to bigger droplets of 5500 to $6500 \mathrm{~nm}$ as were visible in the average. The graphs in Figure 3 represent the average size of droplets in a period of 30 min, thus it was not possible to observe all the intermediate diameters that appeared during this time in those graphs (e.g. diameter of 2000 $\mathrm{nm}$ ). When HFBll and ocimene were mixed, during the first few minutes of the measurement there were monomeric hydrophobins. As time passed, these were no longer visible. Droplets with a size of approximately $2000 \mathrm{~nm}$, which were also present in the pure ocimene sample (though these were unstable and disappeared quickly due to clustering), stayed detectable for a longer period of time.

The evolution of droplet size during 10 days showed the stability of the HFBll-ocimene interaction (Figure 5). After 4 days the results were similar with those of day 1 . After 8 days however, the biggest droplets (from 1000 to $6500 \mathrm{~nm}$ ) were no longer present and instead, the amount of droplets around $600 \mathrm{~nm}$ augmented. This size was also visible in the former samples. A possible explanation for the disappearance of the bigger droplets (corresponding to the micelles of ocimene) is that further enlargement of these droplets resulted in a diameter outside of the detection limit. Due to this, the percentage of smaller droplets that were still present in the solution augmented in the presented curve. It was found that after 10 days, the droplet sizes were still between $230 \mathrm{~nm}$ and $5500 \mathrm{~nm}$.

\section{HFBII migration to the interface}

The migration of HFBll to the surface of a liquid was studied at room temperature with $2 \mathrm{mg} \kappa-\mathrm{HFBII}$ and $0.4 \mu \mathrm{g}$ ocimene in 100 $\mathrm{mL}$ solution. It has been stated before that HFBll is a surface active compound which $100 \mathrm{mg} / \mathrm{L}$ of $\alpha$-grade (hydrophobin after foam fractionation) resulted in dropping off the surface tension of the

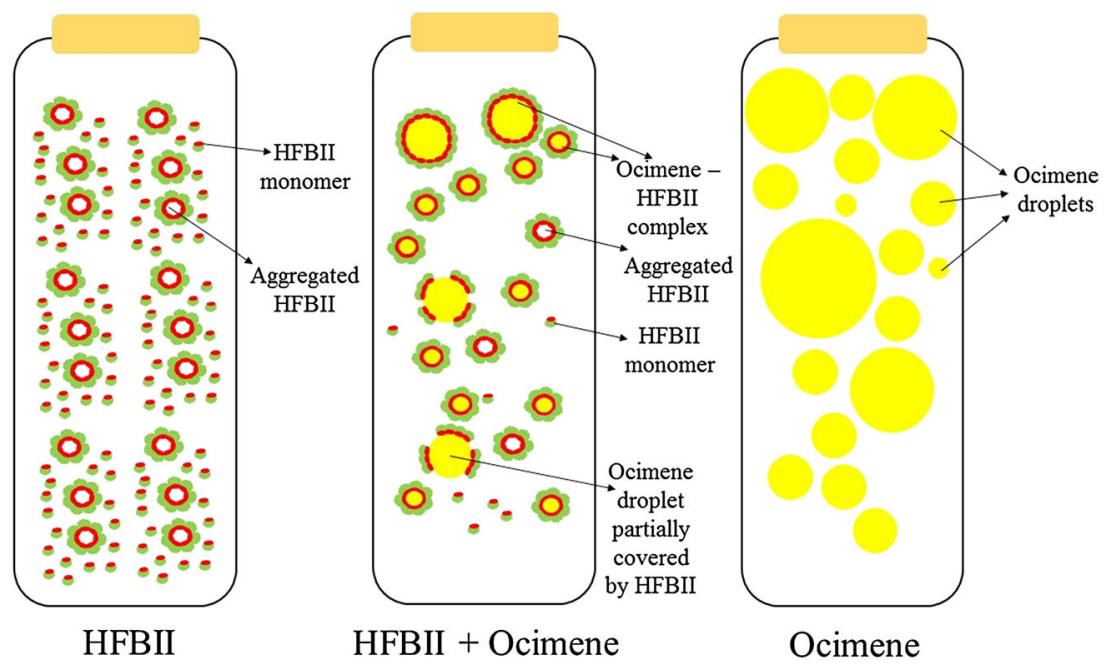

Figure 4. A theoretical model of HFBll, ocimene and the mixture droplets in solution

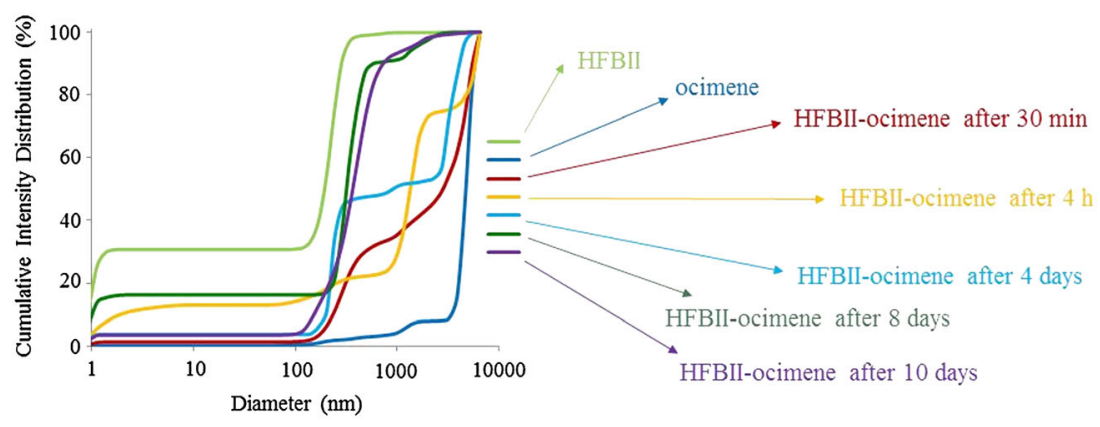

Figure 5. Evolution of the droplet size for the mixture of HFBll and ocimene over a period of 10 days 
water from 72 to $40 \mathrm{~mJ} / \mathrm{m}^{2}$. $^{[31]}$ The amount of HFBII at the top phase $(10 \%)$ of the sample was measured at defined conditions. The migration of HFBll occurred mostly in the first 3 days (Figure 6a). After that, the rate of migration diminished, probably due to the strong emulsion created by the HFBII-ocimene system. Higher temperature caused the migration to be more rapidly (Figure $6 \mathrm{~b}$ ) due to the increased evaporation rate of ocimene. Consequently, destabilization of the ocimene-HFBll complex occurred. The added amount of ocimene played a significant role to alter this rate (Figure $6 \mathrm{c}$ ). A higher amount of ocimene caused a more homogenous distribution of HFBII in the bulk solution. By increasing the amount of ocimene in the bulk from $0.08 \mu \mathrm{g}$ to $0.40 \mu \mathrm{g}$, the hydrophobin migration to the interface was reduced by a factor 1.5. Remarkably, when there was gaseous $\mathrm{CO}_{2}$ present in the system, the migration of HFBll to the interface was much quicker (Figure $6 \mathrm{~d}$ ). This showcases the fact that $\mathrm{CO}_{2}$ has a strong affinity to the hydrophobic patch of HFBII and competes with ocimene to extract hydrophobin from the bulk, preponing the migration of HFBII to the top phase of the liquid. Another parameter that has been examined was the intensity of shaking. Although the rate of vortex at 1000 and 2000 rotations per minute did not significantly influence the HFBII migration rate, when using a magnetic field apparatus to mix HFBII and ocimene, the migration was postponed (Figure $6 \mathrm{e}$ ). This shows that with a strong emulsification process, the solubility of ocimene in the mixture remained for a longer period of time. However, this postponing effect was more noticeable after 1 day than after longer times.

\section{Retention of ocimene}

The previous experiments have given evidence of the interaction between HFBII and ocimene. In this experiment, the longer retention of ocimene in a water solution by the addition of HFBII was examined.
The ocimene concentration was monitored in the solution by DI-SPME-GC-MS. The standard contained both $\beta$-cis and $\beta$-trans ocimene. The total ion chromatogram (TIC) showed $\beta$-cis ocimene and $\beta$-trans ocimene, with retention times $8.65 \mathrm{~min}$ and $8.77 \mathrm{~min}$, respectively (refer to supplementary data). The ratio of cis/trans ocimene in the standard solution was 2.65. The amount of ocimene was obtained as the sum of the area of the isomers in the TIC. The maximum concentration of ocimene that gives a linear response from the detector was found to be $5.9 \times 10^{-6} \mathrm{mg} / \mathrm{mL}$. There is indeed a linear relation between the concentration of ocimene in the vial, and the relative peak area of the ocimene to the internal standard (refer to supplementary data).

The evolution of the ocimene concentration for each sample in function of time is displayed in Figure 7. According to the figure, there was a severe decline of ocimene (with the initial

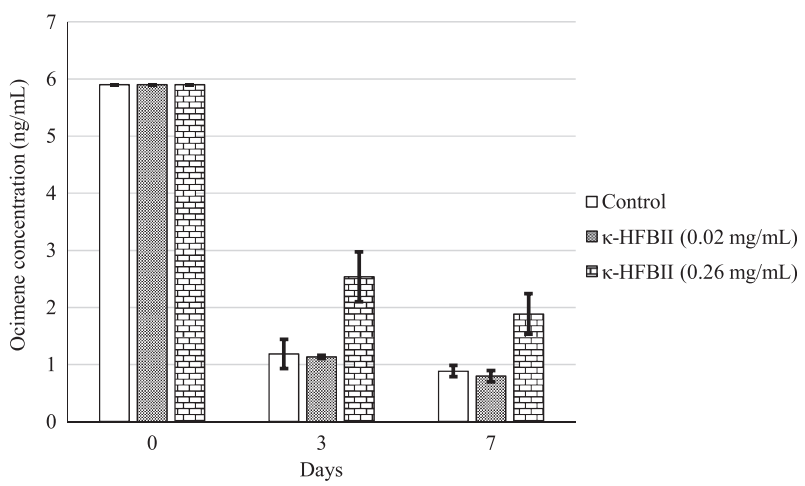

Figure 7. Evolution of ocimene concentration in a solution containing 0 , 0.02 , and $0.26 \mathrm{mg} / \mathrm{mL}$ HFBII during 7 days $(\mathrm{n}=2)$
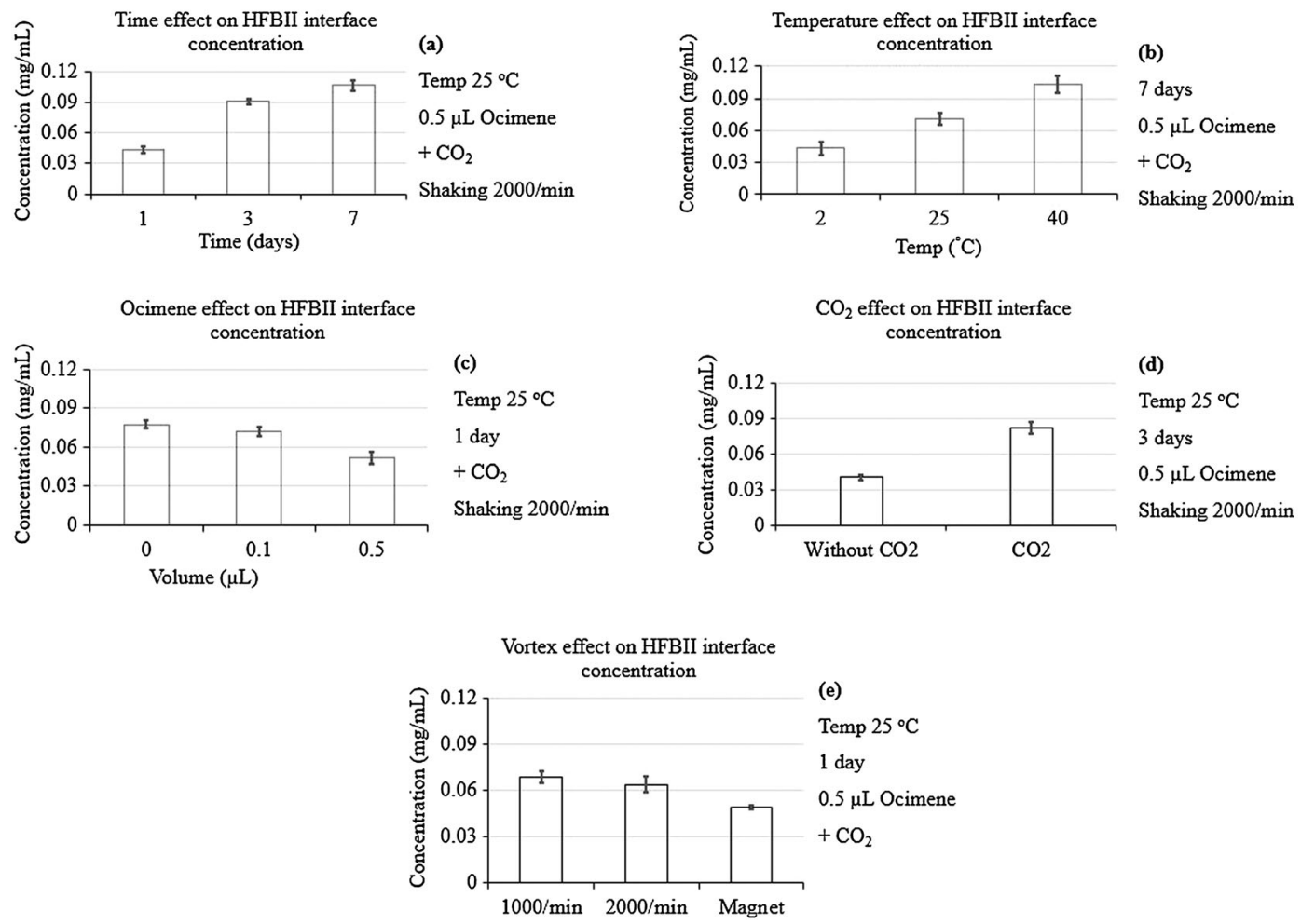

Figure 6. Effects on the migration of HFBll to the interface of the solution according to (a) the time (days), (b) $\mathrm{T}\left({ }^{\circ} \mathrm{C}\right),(\mathrm{c})$ ocimene concentration $(\mu \mathrm{L} / 100 \mathrm{~mL})$, (d) presence of $\mathrm{CO}_{2}$ in the solution, and (e) technique used for shaking the solution. The $y$-axis in all figures represents the concentration of $\mathrm{HFBll}$ ( $\mathrm{mg} / \mathrm{mL}$ ) 
concentration of $5.9 \times 10^{-6} \mathrm{mg} / \mathrm{mL}$ ) in the first 3 days. The decline was however slower for the sample including HFBll with a concentration of $0.26 \mathrm{mg} / \mathrm{mL}$. In other words, after 3 days only $20.1 \%$ of the original ocimene concentration remained in the sample without HFBII (control sample). For the sample with the lowest concentration of HFBII $(0.02 \mathrm{mg} / \mathrm{mL})$, the result was quite similar, $19.2 \%$ of the original ocimene concentration was left. When the HFBII concentration was $0.26 \mathrm{mg} / \mathrm{mL}, 43.0 \%$ of the original ocimene remained after 3 days. From day 3 through 7 , the concentration declined further. From the original ocimene in the control sample and the sample with $0.020 \mathrm{mg} \mathrm{HFBII/mL,} 13.5 \%$ and $15.0 \%$ remained, respectively, though in the sample with $0.26 \mathrm{mg}$ $\mathrm{HFBll} / \mathrm{mL}$, this amount was higher and equal to $32.0 \%$.

It can be stated that the interaction between HFBll and ocimene is at the basis of these observations. The principles of mechanism to prevent loss of ocimene, either in the form of vaporisation or any physico-chemical reactions, are similar. The shells of hydrophobin prevents deterioration. Hydrophobin films can encapsulate the molecules of ocimene. This covering may prevent the escape of ocimene to the gas phase. The hypothesis is that the solubility of ocimene in a solution is increased by emulsification with HFBII. With sufficient HFBII molecules in the system, the difference in ocimene concentration in comparison with a sample without HFBII would be significant and consequently, less ocimene molecules could escape the liquid. Another possibility is that the films around the micelles of ocimene has slowed down any chemical or physical deteriorations, as for instance oxidation phenomenon. The point is that the hydrophobin itself does not have antioxidant activity but in the form of shell, this protects the content inside. As an added value, the shell of hydrophobin might therefore be considered as an antioxidant substrate. It is however very difficult to confirm this hypothesis due to the complexity of oxidized ocimene measurement. In addition, the diffusion coefficient in water for ocimene has been recorded to be $6.52 \times 10^{-10}$ which is around 10000 times less than the diffusion coefficient of this compound in gas phase $\left(5.46 \times 10^{-6}\right)$ showing the strong tendency of ocimene to hydrophobic phases as for instance air. ${ }^{[32]}$ This confirms the fact that the effect of HFBII was rather related to decrease of the migration rate of the ocimene from the water to the air than the prevention of oxidising reaction.

Given these series of information, HFBll has been proposed to be a new aroma pallet. More experiment set-ups have to be performed in order to improve the yield of execution. The first is to find the best correlation between the amount of the target aroma in the samples and the amount of hydrophobin required. The next can be applying this proposal for the stability of the flavours in real products (e.g. beverages or cereals product containing aroma compounds).

From what has been discussed in this work, it can be concluded that HFBII and ocimene interact together. This was first confirmed by the inhibition of gushing when adding ocimene to sparkling water artificially contaminated by HFBll, plus the observation of new droplets when HFBII and ocimene were mixed. In fact, HFBII emulsified the ocimene. On top of this, these new droplets with a diameter larger than HFBII droplets and smaller than the ocimene droplets existed after 10 days showing that this emulsion was relatively stable. The stability of the emulsion is affected by many parameters, e.g. storing time, temperature, concentration ratio of HFBII and ocimene, emulsification technique, and the presence of $\mathrm{CO}_{2}$. On the other hand, it was observed that when HFBII was present in an ocimene solution, after a few days a larger amount of dispersed ocimene still remained in comparison with the sample without HFBII. This indicated that the interaction between HFBII and ocimene slowed down the release of ocimene into the gas phase probably due to the augmented solubility.

The hydrophobicity of hydrophobin is the key to both the negative and the positive functionalities of this protein. To inhibit the negative effect of hydrophobin in industry, it is essential to block the hydrophobic patch(es) of hydrophobins by using attractive components with the potential to interact with this patch. In order to inhibit the gushing phenomenon, it is required to inhibit the interaction between $\mathrm{CO}_{2}$ and the hydrophobic patch(es) of hydrophobins. Therefore, it is needed to find a molecule that can compete with $\mathrm{CO}_{2}$ to occupy the hydrophobic patch(es). This molecule should be apolar and present in adequate amount to neutralize the interaction. There exist many compounds with the described requirements such as linalool, myrcene, ocimene, etc. in carbonated beverages or others in medicines, to interact with hydrophobin. The new complexes may also cause a postponed release of those volatiles. Also, it would be great if another emulsifier, which has already been fully studied, can be used as a comparison to check the emulsifying performance of Class II hydrophobins.

\section{Acknowledgements}

The authors are grateful to the Hydrophobin Chair II - KU Leuven, for supporting this study.

\section{References}

1. A. Seuvre, E. Philippe, S. Rochard, A. Voilley, Food Chem. 2006, 96, 104. 2. M. Terta, G. Blekas, A. Paraskevopoulou. Food Hydrocolloids. 2006, $20,863$. 3. G. Bortnowska. Pol. J. Food Nutr. Sci. 2010, 60, 301.

4. A. Madene, M. Jacquot, J. Scher, S. Desobry. Int. J. Food Sci. Tech. 2006, $41,1$.

5. A. C. Bertolini, A. C. Siani, C. R. F. Grosso. J. Agri. Food Chem. 2001, 49, 780.

6. E. Guichard. Biotechnol. Adv. 2006, 24, 226.

7. M. B. Linder, G. R. Szilvay, T. Nakari-Setälä, M. E. Penttilä. FEMS Microbiol. Rev. 2005, 29, 877.

8. A. Paananen, E. Vuorimaa, M. Torkkeli, M. E. Penttilä, M. Kauranen, O. Ikkala, H. Lemmetyinen, R. Serimaa, M. B. Linder. Biochemistry 2003, $42,5253$.

9. G. R. Szilvay, A. Paananen, K. Laurikainen, E. Vuorimaa, H. Lemmetyinen, J. Peltonen, M. B. Linder. J. Am. Chem. Soc. 2007, 46, 2345.

10. M. Khalesi, S. Deckers, K. Gebruers, L. Vissers, H. Verachtert, G. Derdelinckx. Cerevisia 2012, 37, 3.

11. J. Li, E. M. Perdue, S. G. Pavlostathis, R. Araujo. Environ. Int. 1998, 24, 353.

12. H. Loza-Tavera. Adv. Exp. Med. Biol. 1999, 464, 49.

13. N. Dudareva, L. M. Murfitt, C. J. Mann, N. Gorenstein, N. Kolosova, C. M. Kish, C. Bonham, K. Wood. Plant Cell 2000, 12, 949.

14. J. Patzak, V. Nesvadba, A. Henychova, K. Krofta. Biochem. Sys. Ecol. 2010, 38, 136.

15. S. M. Noe, P. Ciccioli, E. Brancaleoni, F. Loreto, Ü. Niinemets. Atmos. Environ. 2006, 40, 4649.

16. M. Z. Cic, M. Li. Perfumer \& Flavorist 2013, 38, 42.

17. J. Hakanpää, A. Paananen, S. Askolin, T. Nakari-Setälä, T. Parkkinen, M. E. Penttilä, M. B. Linder, J. Rouvinen. J. Biol. Chem. 2004, 279, 534.

18. S. Deckers, T. Venken, M. Khalesi, K. Gebruers, G. Baggerman, Y. Lorgouilloux, Z. Shokribousjein, V. Ilberg, C. Schönberger, J. Titze, H. Verachtert, C. Michiels, H. Neven, J. Delcour, J. Martens, G. Derdelinckx, M. De Maeyer. J. Am. Soc. Brew. Chem. 2012, 70, 249.

19. S. M. Deckers, Y. Lorgouilloux, K. Gebruers, G. Baggerman, H. Verachtert, H. Neven, C. Michiels, G. Derdelinckx, J. A. Delcour, J. Martens. J. Am. Soc. Brew. Chem. 2011, 69, 144.

20. M. Khalesi, Q. Zune, T. Telek, D. Riveros Galan, H. Verachtert, D. Toye, K. Gebruers, G. Derdelinckx, F. Delvigne. Biochem. Eng. J. 2014, 88, 171.

21. Z. Shokribousjein, D. Riveros Galan, C. Michiels, K. Gebruers, H. Verachtert, J. Martens, C. Peeters, G. Derdelinckx, (2015). J. Food Eng. 2015, 145, 10-18.

22. H. Luo, J. Xu, X. Yu. Asia Pac. J. Clin. Nutr. 2007, 16, 286.

23. R. M. Peña, J. Barciela, C. Herrero, S. Garcia-Martin. J. the Sci. Food Agr. 2005, 85, 1227. 
24. T. Neuhof, R. Dieckmann, I. S. Druzhinina, C. P. Kubicek, T. Nakari-Setala, M. Penttila, H. von Dohren. FEBS J. 2007, 274, 841.

25. A. Astoreca, C. Magnoli, C. Barberis, S. M. Chiacchiera, M. Combina, A. Dalcero. Sci. Total Environ. 2007, 388, 16.

26. Z. Shokribousjein, A. Philippaerts, D. Riveros, J. Titze, Y. Ford, S. M. Deckers, M. Khalesi, J. A. Delcour, K. Gebruers, H. Verachtert, V. Ilberg, G. Derdelinckx, B. Sels. J. Am. Soc. Brew. Chem 2014, 72, 12-21.

27. T. Sarlin, T. Nakari-Setälä, M. Linder, M. Penttilä, A. Haikara. J. I. Brewing 2005, 111(2), 105.

28. J. Hakanpää, G. R. Szilvay, H. Kaljunen, M. Maksimainen, M. B. Linder, J. Rouvinen. Protein Sci. 2006, 15, 2129.

29. N. Kameda, N. Sogoshi, S. Nakabayashi. Surf. Sci. 2008, 602, 1579.

30. H. Li, L. Hu, Z. Xia. Materials. 2013, 6, 3676.
31. M. Khalesi, N. Mandelings, Z. Shokribousjein, D. Riveros-Galan, H. Verachtert, K. Gebruers, F. Delvigne, I. Vankelecom, G. Derdelinckx. Cerevisia 2014, 38, 129.

32. Ü. Niinemets, M. Reichstein, M. Staudt, G. Seufert, J. D. Tenhunen. Plant Physiol. 2002, 130, 1371.

\section{Supporting information}

Additional supporting information may be found in the online version of this article at the publisher's web site 